\title{
General Psychiatry How psychiatrists coordinate treatment for COVID-19: a retrospective study and experience from China
}

To cite: Yue L, Wang J, Ju M, et al. How psychiatrists coordinate treatment for COVID-19: a retrospective study and experience from China. General Psychiatry 2020;33:e100272. doi:10.1136/ gpsych-2020-100272

- Additional material is published online only. To view please visit the journal online (http://dx.doi.org/10.1136/ gpsych-2020-100272).

$\mathrm{LY}$ and JW are joint first authors.

Received 16 May 2020

Revised 11 June 2020

Accepted 24 June 2020

Check for updates

(C) Author(s) (or their employer(s)) 2020. Re-use permitted under CC BY-NC. No commercial re-use. See rights and permissions. Published by BMJ.

For numbered affiliations see end of article.

Correspondence to

Yifeng Shen;

shenyifeng@yahoo.com

\section{ABSTRACT}

Background Patients with COVID-19 are at high risk of developing mental health problems; however, the prevalence and management of mental disorders and how psychiatrists coordinate the treatment are unclear.

Aims We aimed to investigate the mental health problems of patients infected with COVID-19 and to identify the role of psychiatrists in the clinical treatment team during the pandemic. We also share the experience of psychiatric consultations of patients with COVID-19 in Shanghai, China.

Methods We analysed data from the psychiatric medical records of 329 patients with COVID-19 in the Shanghai Public Health Clinical Center from 20 January to 8 March 2020. We collected information including sociodemographic characteristics, whether patients received psychiatric consultation, mental health symptoms, psychiatric diagnoses, psychiatric treatments and severity level of COVID-19.

Results Psychiatric consultations were received by $84(25.5 \%)$ patients with COVID-19. The most common symptoms of mental health problems were sleep disorders $(75 \%)$, anxiety $(58.3 \%)$ and depressive symptoms (11.9\%). The psychiatric consultation rate was highest in critically ill patients $(69.2 \%)$, with affective symptoms or disturbed behaviour as their main mental health problems. Psychiatric diagnoses for patients who received consultation included acute stress reaction (39.3\%), sleep disorders (33.3\%), anxiety (15.5\%), depression (7.1\%) and delirium (4.8\%). In terms of psychiatric treatments, $86.9 \%$ of patients who received psychiatric consultation were treated with psychotropic medications, including non-benzodiazepine sedative-hypnotic agents (54.8\%), antidepressants (26.2\%), benzodiazepines (22.6\%) and antipsychotics (10.7\%). Among the 76 patients who were discharged from the hospital, $79 \%$ had recovered from mental health problems and were not prescribed any psychotropic medications. The symptoms of the remaining $21 \%$ of patients had improved and they were prescribed medications to continue the treatment.

Conclusions This is the first study to report psychiatric consultations for patients with COVID-19. Our study indicated that a considerable proportion of patients with COVID-19, especially critically ill cases, experienced mental health problems. Given the remarkable effect of psychiatric treatments, we recommend that psychiatrists be timely and actively involved in the treatment of COVID-19.

\section{INTRODUCTION}

COVID-19 has been prevalent in China since December 2019 and is now spreading all over the world. As the pandemic spreads, some people with mental health disorders have been infected by COVID-19. ${ }^{1}$ In addition, over $50 \%$ of patients with COVID-19 had anxiety and depression symptoms according to a previous survey. ${ }^{2}$ Moreover, critically ill cases may develop delirium, involving visual hallucination and disturbed behaviour, which can interfere with their treatment. Hence, psychiatrists are needed to alleviate patients' mental symptoms and to prevent extreme events such as suicide and impulsive behaviour. $^{3}$

It is known to all that a major incident can lead to psychological stress. In the Mental Health Gap Action Programme Intervention Guide, the WHO indicated that it was necessary to provide psychological first aid for persons in distress who had just experienced traumatic events. ${ }^{4}$ Mental crisis intervention, as a kind of urgent and rapid psychological intervention, aims to ease the person's mental pain and stabilise their emotions immediately. Mental crisis intervention can prevent and reduce mental impairment caused by stress or traumatic events, such as acute stress disorder or post-traumatic stress disorder (PTSD). Therefore, in Shanghai, a remote consultation network was built for timely psychological crisis intervention. ${ }^{5}$ Moreover, at the early stage of the COVID-19 outbreak (23 January 2020), psychiatrists from Shanghai Mental Health Center were integrated with the multiple disciplinary team (MDT) to jointly fight the outbreak.

Facing this critical situation, patients with COVID-19 are at high risk of developing mental health problems. To our knowledge, there has been no report on psychiatric management and outcomes of patients with COVID-19. This study is a retrospective 


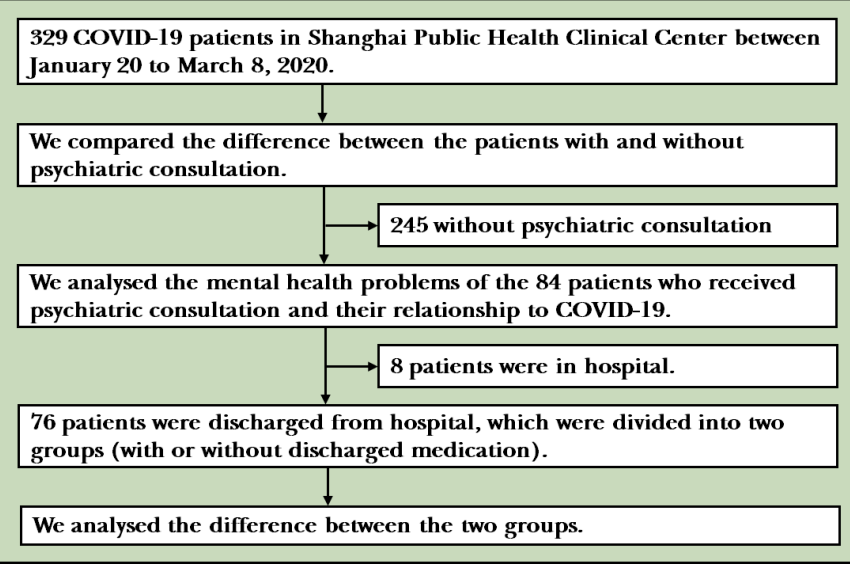

Figure 1 Study flowchart.

analysis of psychiatric medical records of 329 patients with COVID-19 in the Shanghai Public Health Clinical Center. We aimed to evaluate the mental health problems of patients with COVID-19 and to explore the synergic effect of involving psychiatrists in their treatment. We also aimed to share the experience of psychiatric consultations and management of patients with COVID-19 in Shanghai, China.

\section{METHODS}

\section{Participants and study design}

We retrospectively collected data from medical records of patients with a confirmed COVID-19 diagnosis, especially records on psychiatric consultation and psychotropic medication. All patients were diagnosed and their severity levels were defined according to the expert consensus statement on the comprehensive treatment and management of COVID-19 from the Shanghai municipality. ${ }^{6}$ The data from a total of 329 patients with COVID-19 were pulled from the Shanghai Public Health Clinical Center, where patients were hospitalised from 20 January to 8 March 2020. The sample consisted of 171 men and 158 women. Ages ranged from 15 to 88 years, with a mean (SD) age of 49.78 (16.14) years. Figure 1 shows the study flowchart.

All psychiatric diagnoses were made by psychiatrists using the diagnostic criteria of the International Classification of Diseases, 10th edition. Patients were referred to psychiatrists in two ways. Psychiatrists were in the hospital 24 hours every day and regularly performed ward rounds in order to identify mental health problems among patients. Additionally, psychiatrists provided consultation at any time when physicians found any psychiatric or psychological problems (eg, hallucinations in patients in the intensive care unit (ICU)).

\section{Statistical analysis}

We compared the demographic information of patients who received psychiatric consultations and those who did not. The relationships between use of psychiatric consultation and severity level of COVID-19 as well as age groups were analysed. Group comparisons of the demographic and clinical data were conducted using two-tailed independent samples t-tests for continuous variables and $\chi^{2}$ tests for dichotomous variables with SPSS V.19.0. The significance level was set at $\mathrm{p}<0.05$ and all tests were two-tailed.

\section{RESULTS}

\section{Demographic characteristics}

Of the 329 patients with COVID-19, 84 (25.5\%) received psychiatric consultation. There were more women in those who received psychiatric consultation $(59.5 \%)$ than those who did not $(44.1 \%)\left(\chi^{2}=5.976, \mathrm{p}=0.015\right)$. Hospitalisation days were also longer in the former group. There was no significant difference in age between the two groups (see table 1).

\section{Clinical manifestation}

The three most common symptoms of mental illness were sleep disorders $(75 \%)$, anxiety symptoms $(58.3 \%)$ and depressive symptoms (11.9\%) (see table 2).

The percentage of those receiving psychiatric consultation was different among the three severity levels of COVID-19 $\left(\chi^{2}=12.492, p=0.002\right)$. Over half of critically ill patients needed psychiatric consultation (9 cases, $69.2 \%$ ). There was no significant difference in the use of psychiatric consultation among the different age groups (see table 3).

\section{Psychiatric diagnosis}

The psychiatric diagnoses for the 84 patients with COVID-19 who used mental health consultation were as follows: acute stress reaction (39.3\%), sleep disorders $(33.3 \%)$, anxiety state $(15.5 \%)$, depression state or major depression $(7.1 \%)$, and delirium (4.8\%) (see table 4). Among the 84 patients, 3 had a history of mental disorder (2 depression and 1 schizophrenia), all of whom received long-term psychiatric medication treatment and their mental state was stable before the COVID-19 breakout. In addition, a patient with vascular dementia but without behavioural or psychological symptoms before admission was diagnosed with dementia comorbid with delirium. Among the four patients diagnosed with delirium, three were critically ill and one had mild/moderate COVID-19 symptoms. The psychiatric diagnoses of the nine critically ill patients were anxiety (three cases), acute stress reaction (two cases), depressive state (one case), vascular dementia comorbid with delirium (one case), schizophrenia comorbid with delirium (one case), and delirium (one case).

\section{Psychiatric intervention}

Eleven $(13.1 \%)$ out of 84 patients were given supportive psychotherapy, and their mental state improved after therapy. The rest $(73,86.9 \%)$ of the patients were treated with psychotropic medications in addition to psychotherapy. Among these patients, 22 (26.2\%) were prescribed antidepressants, 19 (22.6\%) used benzodiazepines, 9 $(10.7 \%)$ used antipsychotic drugs, and $46(54.8 \%)$ used 
Table 1 Comparison of the demographic characteristics between the two groups

\begin{tabular}{|c|c|c|c|c|}
\hline Characteristics & $\begin{array}{l}\text { With psychiatric } \\
\text { consultation }(n=84)\end{array}$ & $\begin{array}{l}\text { No psychiatric } \\
\text { consultation }(n=245)\end{array}$ & $t / \chi^{2}$ & $P$ value \\
\hline Gender, male/female (\%) & $34 / 50(40.5)$ & 137/108 (55.9) & 5.976 & 0.015 \\
\hline Hospital stay, mean (SD), days ${ }^{*}$ & $19.53(8.23)$ & $16.54(6.81)$ & 2.858 & 0.005 \\
\hline Hospital stay, mean (SD), days, all† & $21.06(9.45)$ & $17.10(7.41)$ & 3.490 & $<0.001$ \\
\hline
\end{tabular}

*Patients discharged: with psychiatric consultation group: 76 cases; no psychiatric consultation group: 234 cases; only for discharged patients.

†The number of days for patients still hospitalised was calculated until 8 March.

ICU, intensive care unit.

non-benzodiazepine sedative-hypnotics (zolpidem). The antidepressants included sertraline (14 of 22), Deanxit (6 of 22), escitalopram (1 of 22) and fluvoxamine (1 of 22). In terms of antipsychotic drugs, eight out of nine patients used olanzapine and one used quetiapine.

Psychotropic medication treatment followed the principle of short-term, low-dose and gradual titration according to the patient's condition. During the follow-up before discharge, no serious adverse events had occurred. All of the patients who received psychiatric consultation were offered supportive psychotherapy. Among the 76 patients who were discharged from the hospital, 16 were prescribed psychiatric drugs according to their discharge record, including 6 cases with hypnotic sedative drugs, 4 with antidepressants, 4 with hypnotic sedative drugs combined with antidepressants, and 2 with low-dose antipsychotic drugs. The rest $(60,78.9 \%)$ of the patients recovered well and the dosage of their drugs was gradually tapered during hospitalisation without discomfort. The patients with and without discharge medications showed insignificant differences in age, gender and hospital stay. However, the distribution of the psychiatric diagnoses was different $(p<0.001)$. Those who were diagnosed with major depression needed psychiatric drugs after being out of hospital, and the patient with delirium did not need to continue psychiatric treatment.

Table 2 Psychological symptoms of patients receiving psychiatric consultation

\begin{tabular}{|c|c|}
\hline Psychological symptoms & $\begin{array}{l}\text { Patients receiving } \\
\text { psychiatric } \\
\text { consultation, n (\%) }\end{array}$ \\
\hline $\begin{array}{l}\text { Sleep disorders (insomnia, early } \\
\text { awakening, difficulty falling } \\
\text { asleep) }\end{array}$ & $65(75.5)$ \\
\hline Anxiety (tension, worry and so on) & $50(58.1)$ \\
\hline $\begin{array}{l}\text { Depression (negative and } \\
\text { pessimistic, and so on) }\end{array}$ & $10(11.6)$ \\
\hline $\begin{array}{l}\text { Inexplicable somatic discomfort } \\
\text { symptoms }\end{array}$ & $9(10.4)$ \\
\hline Wrangle, disturbed behaviour & $5(5.8)$ \\
\hline Hallucination and delusion & $3(3.4)$ \\
\hline
\end{tabular}

\section{DISCUSSION}

Main findings

This study summarised the psychiatric characteristics and treatment of patients with COVID-19 in the Shanghai Public Health Clinical Center (the only hospital that treats adult patients with COVID-19 in Shanghai). The results showed a high prevalence of mental and psychological problems among patients with COVID-19. Since most patients achieved satisfactory mental health outcomes after psychiatric consultation and targeted treatment, it is reasonable to conclude that psychiatrists promoted their recovery by cooperating with the MDT in fighting against COVID-19.

Facing this pandemic situation, the general population in China exhibited marked psychological stress responses, ${ }^{7}$ and patients with confirmed COVID-19 were more prone to experience psychological stress and even mental breakdown. In this study, $25.5 \%$ of patients with COVID-19 received psychiatric consultations, suggesting a high risk of developing mental or psychological diseases.

Table 3 Use of psychiatric consultation among different severity levels of COVID-19 and age groups

\section{Patients receiving \\ psychiatric \\ consultation/all \\ patients in different \\ groups}

$\chi^{2} \quad$ P value

Use of psychiatric consultation among different severity levels of COVID-19

$\begin{array}{lccc}\begin{array}{l}\text { Mild/moderate } \\ (\%)\end{array} & 73 / 301(24.3) & 12.492 & 0.002 \\ \text { Severe (\%) } & 2 / 15(13.3) & & \\ \text { Critically ill (\%) } & 9 / 13(69.2) & & \end{array}$

Use of psychaitric consultation among different age groups

$\begin{array}{llll}\text { Young, } 15-39 & 22 / 110(20.0) & 3.111 & 0.21\end{array}$

years (\%)

Middle, 40-54 23/74 (31.1)

years (\%)

Older, $\geq 55$ years $39 / 145(26.9)$

(\%) 


\begin{tabular}{ll}
\hline Table $4 \quad$ Psychiatric diagnosis & \\
\hline Psychiatric diagnosis & Cases (n=84) \\
\hline Acute stress reaction (\%) & $33(39.3)$ \\
Sleep disorders (\%) & $28(33.3)$ \\
Anxiety state (\%) & $13(15.5)$ \\
Major depression (\%) & $2(2.4)$ \\
Depressive state (\%) & $4(4.7)$ \\
Delirium (\%) & $2(2.4)$ \\
Dementia with delirium (\%) & $1(1.2)$ \\
Schizophrenia with delirium (\%) & $1(1.2)$ \\
\hline
\end{tabular}

The most common psychiatric diagnoses were acute stress disorders $(39.3 \%)$, sleep disorders $(33.3 \%)$ and anxiety $(15.5 \%)$, and several patients developed major depression $(7.1 \%)$. Our study was consistent with a previous psychological survey reporting symptoms of depression and anxiety among more than half of patients with COVID-19 in Wuhan. ${ }^{2}$ It is noteworthy that the prevalence of mental health problems may have been underestimated because some patients might experience psychological difficulties but did not ask for psychiatric consultation. A screening scale, such as the Patient Health Questionnaire, 9-item version, ${ }^{8}$ is needed to identify psychological or psychiatric problems in every patient with COVID-19 in the future. Nevertheless, our data support that patients with COVID-19 had a definite demand for mental health services, and early intervention and professional treatment are needed urgently. ${ }^{9}$

The role of mental crisis intervention after a major public health incident is becoming increasingly important. Unlike the 'post-disaster' intervention, our psychiatrists have been working in isolation wards at the initial stage of the epidemic and continuously stayed in the hospital from the third day of hospitalisation of the first patient with COVID19 , psychiatrists have never been absent. As the core of crisis intervention, psychiatrists not only conducted psychological intervention, but also prescribed psychotropic drugs when necessary to deal with depressive problems, panic attacks and organic psychosis (including delirium). Studies have shown that early intervention can reduce psychiatric symptoms and the risk of developing PTSD ${ }^{10}$ For patients with COVID-19, psychiatric treatment should focus on controlling symptoms rapidly, improving sleep, ensuring rest and early intervention for depression. Our study showed that $87 \%$ of patients who received psychiatric consultation were prescribed medication (mostly sedative), which may be inconsistent with Australian guidelines for acute stress therapy that state "medication is not recommended except for severe depression that cannot be alleviated by psychotherapy'. ${ }^{11}$ However, we are facing a very different situation: (1) the rapid control of psychiatric symptoms is important to efficiently treat COVID-19; (2) conducting regular psychotherapy in isolated wards with protective clothing is difficult, and (3) the impulsive behaviour presented by some patients may influence health outcomes and treatment, and even increase the risk of medical staff's exposure to the virus. Hence, after careful consideration, drug treatment was considered as an important choice in this special situation. Among all the drugs, antidepressants and sedatives were the most prescribed. For major depression, besides psychological crisis intervention, antidepressants combined with sedative-hypnotic drugs were effective. ${ }^{10}$ The psychiatrists adjusted the psychological intervention or medication according to the patient's condition every day. Most patients' medications were gradually reduced or discontinued as their symptoms improved, and no serious adverse events occurred, indicating the effectiveness and safety of this practice. We provided the details of treatment for three typical cases in the online supplementary material. WHO Director-General Dr Tedros emphasised that 'We must remember that these are people, not numbers.' The effort made by psychiatrists is helpful to show that 'people are what matters'. Early psychiatric intervention and positive treatment can assist the medical team in managing the pandemic.

Recently, the psychiatric consultation rate in general hospitals has been growing rapidly. Studies have shown that there is the greatest demand for consultation in people with behavioural disorders and emotional symptoms. ${ }^{12}$ It could be difficult for doctors who do not specialise in mental health to deal with such symptoms, so consultation-liaison psychiatry (CLP) has attracted great attention. ${ }^{13}$ In this study, there were significant differences in psychiatric characteristics between critically ill patients and mild/moderate patients. The percentage of use of psychiatric consultation for the former was higher ( $69.2 \%$ vs $24.3 \%$ ), reflecting that the more serious COVID-19 is, the greater the possibility of comorbid mental health problems. Previous studies have also reported that psychiatric consultation is most common in ICU wards in general hospitals. ${ }^{13}$ Among the nine critically ill patients who received psychiatric consultation, six cases had affective disorders and three were diagnosed with delirium, which were the two most common reasons for psychiatric consultation. ${ }^{14}$ Low-dose psychotropic medication can effectively alleviate behavioural disorders caused by delirium. ${ }^{15}$ It should be pointed out that, unlike the CLP in general hospitals, the MDT can comprehensively consider the interrelationship between psychosocial factors, physical diseases, mental disorders and medications, and then determine an individualised treatment plan through joint discussion. This model can also facilitate psychiatric knowledge in doctors from other departments. Taken together, psychiatrists have made great contributions to the treatment of patients with COVID-19 in Shanghai through participating in the whole course of treatment in the isolation ward and using effective psychotherapy and psychiatric medications. Although modification and improvement are needed, our collaborative work model is well worth considering in the future of COVID-19 treatment.

In addition, maintaining the mental health of front-line staff was also a particularly important task for psychiatrists 
during the epidemic. The psychiatrists in the team, as the 'guardians of the mind', used electronic questionnaires to assess the mental health risks of medical staff and dealt with various psychological stress at any time, such as insomnia and anxiety. They also provided small lectures, individual psychological counselling, stress reduction training and mindfulness treatment for medical staff, which all received positive feedback.

\section{Limitations}

The study has some limitations. First, this is a retrospective analysis, not a prospective study. This could underestimate the burden of psychiatric conditions, and thus cannot fully evaluate the effect of mental health services in patients with COVID-19. Second, the study took place in only one hospital and included a relatively small number of participants. Nevertheless, we believe that the study provides valuable information and discussion on this global public health event.

\section{Implications}

There is a definite demand for mental health services in patients with COVID-19, especially in critically ill cases. The strategy to integrate psychiatrists into the medical team to fight COVID-19 can benefit the mental health of both the patients and the front-line medical staff. In order to mitigate the impact of this worldwide pandemic, we recommend that psychiatrists be actively involved in the treatment of COVID-19.

\section{Author affiliations}

${ }^{1}$ Department of Psychiatry, Shanghai Jiao Tong University School of Medicine, Affiliated Shanghai Mental Health Center, Shanghai, China

${ }^{2}$ Department of Social Medicine, School of Public Health, Fudan University, Shanghai, China

${ }^{3}$ Department of Population Science and Experimental Medicine, University College London, London, UK

${ }^{4}$ Department of Psychiatry, Nanhui Mental Health Center of Pudong New Area, Shanghai, China

${ }^{5}$ Department of General Adult Psychiatry, Xuhui District Mental Health Center, Shanghai, China

${ }^{6}$ Department of Psychiatry, Hongkou District Mental Health Center, Shanghai, China

Contributors LY wrote the draft and made revisions. JW, MJ, YZ, LC, LS, BS and $\mathrm{JC}$ reviewed the article and made revisions. YFS made the final revision. All authors were involved in study design, conduct of the study, analysis of data, interpretation of results or writing the report.

Funding This work was supported by Shanghai Clinical Research Center for Mental Health, Shanghai, China (SCRC-MH (19MC1911100)).

Competing interests None declared.
Patient consent for publication Not required.

Ethics approval The study protocol was approved by the Ethical Committee of the Shanghai Mental Health Center (2020-31).

Provenance and peer review Not commissioned; externally peer reviewed.

Data availability statement Data used in the current manuscript are available from the corresponding author upon request from any qualified investigator.

Open access This is an open access article distributed in accordance with the Creative Commons Attribution Non Commercial (CC BY-NC 4.0) license, which permits others to distribute, remix, adapt, build upon this work non-commercially, and license their derivative works on different terms, provided the original work is properly cited, appropriate credit is given, any changes made indicated, and the use is non-commercial. See: http://creativecommons.org/licenses/by-nc/4.0/.

\section{ORCID iDs}

Ling Yue http://orcid.org/0000-0001-6476-0189

Jun Chen http://orcid.org/0000-0002-3837-2761

Yifeng Shen http://orcid.org/0000-0002-3298-6682

\section{REFERENCES}

1 Yao H, Chen J-H, Xu Y-F. Patients with mental health disorders in the COVID-19 epidemic. Lancet Psychiatry 2020;7:e21.

2 Zhao Q, Hu C, Feng R, et al. Anxiety, depression and somatic symptoms in COVID-19 patients. J Clin Neurol 2020;53:E003.

3 Murthy S, Gomersall CD, Fowler RA. Care for critically ill patients with COVID-19. JAMA 2020;323:1499.

4 Bisson J, Lewis C. Systematic review of psychological first aid, 2009.

5 Jiang X, Deng L, Zhu Y, et al. Psychological crisis intervention during the outbreak period of new coronavirus pneumonia from experience in Shanghai. Psychiatry Res 2020;286:112903.

6 Shanghai Clinical Treatment expert Group for Corona Virus Disease 2019. Comprehensive treatment and management of corona virus disease 2019: expert consensus statement from Shanghai City. Clinical Journal of Infect Disorder 2020;38:134-8.

7 Qiu J, Shen B, Zhao M, et al. A nationwide survey of psychological distress among Chinese people in the COVID-19 epidemic: implications and policy recommendations. Gen Psychiatr 2020;33:e100213.

8 Guo Q, Zheng Y, Shi J, et al. Immediate psychological distress in quarantined patients with COVID-19 and its association with peripheral inflammation: a mixed-method study. Brain Behav Immun 2020. doi:10.1016/j.bbi.2020.05.038. [Epub ahead of print: 19 May 2020] (Published Online First: 19 May 2020).

9 Duan L, Zhu G. Psychological interventions for people affected by the COVID-19 epidemic. Lancet Psychiatry 2020;7:300-2.

10 Hartshorn MA. Crisis intervention Handbook: assessment, treatment, and research. J Am Med Assoc 2001;286:1769-70.

11 Forbes D, Creamer M, Phelps A, et al. Australian guidelines for the treatment of adults with acute stress disorder and post-traumatic stress disorder. Aust N Z J Psychiatry 2007;41:637-48.

12 Wise TN. Update on consultation-liaison psychiatry (psychosomatic medicine). Curr Opin Psychiatry 2008;21:196-200.

13 Vulser H, Vinant V, Lanvin V, et al. Association between the timing of consultation-liaison psychiatry interventions and the length of stay in general Hospital. Br J Psychiatry 2019:1-6.

$14 \mathrm{Ji}$ J, Ye C. Consultation-Liaison psychiatry in China. Shanghai Arch Psychiatry 2012;24:124-30.

15 Chinese Society of Psychiatry, Association CP. Psychopharmacology therapy recommendations for patients with the COVID-19. J Clin Psychiat 2020;53:84-8.

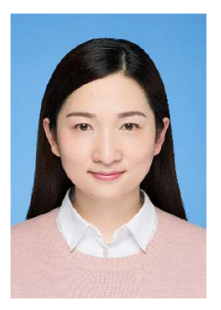

Ling Yue obtained a bachelor's degree of clinical medicine from Shanghai Second Medical University in 2005, a master's degree of Medicine from Shanghai Jiao Tong University School of Medicine in 2013, and a doctorate in mental health from Shanghai Jiao Tong University School of Medicine in 2019. Since 2005, she has been working as a psychiatrist at the Shanghai Mental Health Center, Shanghai, China. She is currently working as an associate chief physician in the Department of Geriatric Psychiatry at Shanghai Mental Health Center, Shanghai, China. Her main research interests include the neuro-imaging and clinical characteristics of cognitive and affective disorders of the elderly. 